\title{
SYNTHESIS AND STRUCTURAL ELUCIDATION OF AMINO ACETYLENIC AND THIOCARBAMATES DERIVATIVES FOR 2-MERCAPTOBENZOTHIAZOLE AS ANTIMICROBIAL AGENTS
}

\author{
ASEEL ALSARAHNI ${ }^{1}$, ZUHAIR MUHI-ELDEEN ${ }^{2}$, ELHAM AL-KAISSI ${ }^{*}$, IBRAHIM AL-ADHAM ${ }^{1}$, NAJAH AL-MUHTASEB ${ }^{2}$ \\ 1Depart. of Pharmaceutics and Pharmaceutical Technology, Faculty of Pharmacy, University of Petra, Amman, Jordan, ${ }^{2}$ Department of \\ Medicinal Chemistry and Pharmacognosy, Faculty of Pharmacy, University of Petra, Amman, Jordan \\ Email: ealkaissi@uop.edu.jo \\ Received: 19 Oct 2016 Revised and Accepted: 21 Dec 2016
}

ABSTRACT

Objective: To design and synthesize amino acetylenic and thiocarbonate of 2-mercapto-1,3-benthiazoles as potential antimicrobial agents.

Methods: A new series of 2-\{[4-(t-amino-1-yl) but-2-yn-1-yl] sulfanyl\}-1,3-benzothiazole derivatives (AZ1-AZ6), and S-1,3-benzothiazol-2-yl-0alkyl carbonothioate derivatives were synthesised, with the aim that the target compounds show new and potential antimicrobial activity. The elemental analysis was indicated by the EuroEA elemental analyzer, and biological characterization was via IR, ${ }^{1} \mathrm{H}$-NMR, [13]C-NMR, DSC were determined with the aid of Bruker FT-IR and Varian $300 \mathrm{MHz}$ spectrometer using DMSO-d 6 as a solvent. In vitro antimicrobial activity, evaluation was done for the synthesised compounds, by agar diffusion method and broth dilution test. The minimum inhibitory concentration (MIC) and the minimum bactericidal concentration (MBC) were determined.

Results: The IR, ${ }^{1} \mathrm{H}-\mathrm{NMR},{ }^{13} \mathrm{C}-\mathrm{NMR}$, DSC and elemental analysis were consistent with the assigned structures. Compound of $2-\{[4-(4-$ methylpiperazin-1-yl)but-2-yn-1-yl] sulfanyl\}-1,3-benzothiazole (AZ1), 2-\{[4-(2-methylpiperidin-1-yl)but-2-yn-1-yl]sulfanyl\}-1,3-benzothiazole (AZ2), 2-\{[4-(piperidin-1-yl) but-2-yn-1-yl]sulfanyl\}-1, 3-benzothiazole (AZ6), S-1,3-benzothiazol-2-yl-0-ethyl carbonothioate (AZ7), and S-1,3benzothiazol-2-yl-0-(2-methylpropyl) carbonothioate (AZ9) showed the highest antimicrobial activity against Pseudomonas aeruginosa (P. aeruginosa), AZ-9 demonstrated the highest antifungal activity against Candida albicans (C. albicans), with MIC of $31.25 \mu \mathrm{g} / \mathrm{ml}$.

Conclusion: These promising results promoted our interest to investigate other structural analogues for their antimicrobial activity further.

Keywords: 2-Mercaptobenzothiazole (2-MBT), Aminoacetylenic, Antimicrobial, Antibacterial, Mannich reaction

(c) 2017 The Authors. Published by Innovare Academic Sciences Pvt Ltd. This is an open access article under the CC BY license (http://creativecommons.org/licenses/by/4. 0/) DOI: http://dx.doi.org/10.22159/ijpps.2017v9i2.15760

\section{INTRODUCTION}

Many species of bacteria are becoming resistant to different types of antimicrobial agents, such as $\beta$-lactam antibiotics, macrolides, quinolones and vancomycin [1, 2]. So the development of new antibacterial and antifungal agents with new biochemical pathways is becoming an urgent demand [3]. Molecules with mercaptobenzothiazole (BTA) moiety have different biological activities [4]. Accordingly, 2-Mercaptobenzothiazole (2-MBT) derivatives have a wide variety of applications [5]. 2-MBT consists of Benzothiazole which is a heterocyclic compound, includes benzene ring fused with 4,5 positions of thiazole ring [6, 7], and the Mercapto (thiol group) substituent at position 2 of thiazole ring that gives the compound antibacterial and anti-inflammatory activity [8]. El-Shaaer et al. (1998) have prepared novel compounds containing MBT linked with chroman4-one moiety (fig. 1). They were screened for antimicrobial activity, against Gram-positive bacteria namely Staphylococcus aureus ( $S$. aureus), Bacillus subtilis (B. subtilis), and Mycobacterium tuberculosis (M. tuberculosis), C. albicans and Saccharomyces cerevisiae (S. cerevisiae). The results showed that 2-MBT derivatives with substituents 6-Cl or 6,7-Dimethyl group on the chroman-4-one moiety increase the compound antimicrobial activity against $S$. aureus, $B$. subtilis, M. tuberculosis, C. albicans and S. cerevisiae [9].

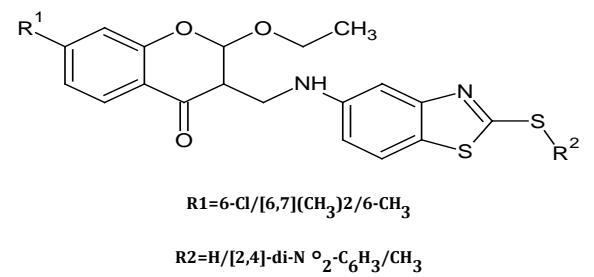

Fig. 1: The structure of substituted 2-MBT with chroman-4-one moiety derivatives (El-Shaaer et al. 1998) [9]
Novel 4-substitut-phenyl-3-chloro-1-[(benzothiazolythio) acetamidyl]2-azetidinone derivatives were synthesized (fig. 2). These novel compounds were screened for their antibacterial and antifungal activities, against $S$. aureus, Aspergillus niger (A. niger) and $C$. albicans. The study showed that compounds derivatives having 3$\mathrm{OH}-\mathrm{C}_{6} \mathrm{H}_{4}$ and $\mathrm{Cl}-\mathrm{C}_{6} \mathrm{H}_{4}$ groups promoted the activity against Candida, while compounds derivatives having $4-\mathrm{OH}-\mathrm{C}_{6} \mathrm{H}_{4}$ and $2-\mathrm{Cl}^{-} \mathrm{C}_{6} \mathrm{H}_{4}$ on position 4 in azetidinone nucleus are very effective against $S$. aureus and A. niger [10].<smiles>[R]OC(=O)CSc1nc2ccc(CC(C)=O)cc2s1</smiles>

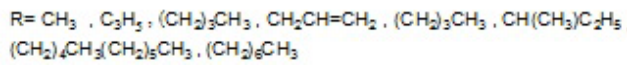

$\mathbf{R}=$

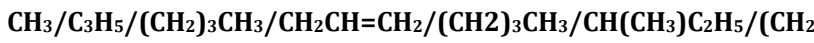
)$_{4} \mathrm{CH}_{3}\left(\mathrm{CH}_{2}\right)_{5} \mathrm{CH}_{3} /\left(\mathrm{CH}_{2}\right)_{6} \mathrm{CH}_{3}$

Fig. 2: The structure of 4-substitut-phenyl-3-chloro-1[(benzothiazolythio) accetamidyl]-2-azetidinone derivatives [10]

In this study, we envision a unique and new series of 2mercaptobenzothiazole derivatives, namely $2-\{[4-(\mathrm{t}-\mathrm{amino}-1-\mathrm{yl})$ but-2-yn-1-yl] sulfanyl\}-1,3-benzothiazole derivatives (AZ1-6), and S-1,3-benzothiazol-2-yl-0-alkyl carbonothioate derivatives (AZ7-9), based on the concept of the fractional based analysis, with the following unique substitutes. In 2-\{[4-(t-amino-1-yl)but-2-yn-1-yl] 
sulfanyl\}-1,3-benzothiazole derivatives, 2-mercaptobenzothiazole ring as a directing moiety towards different sites in bacteria and fungi that lead to antimicrobial activity, amino acetylenic increases the effect, and acts as link providing the following binding interaction: The cyclic amino group provides ionic bonding with their corresponding groups on receptors in bacteria and fungi. Acetylenic group for electrostatic interaction, and the 2-butynyl to provide the appropriate distance between 2-mercaptobenzothiazole moiety and the cyclic amine. In S-1,3-benzothiazol-2-yl-0-alkyl carbonothioate derivatives, the 2-mercaptobenzothiazole moiety promotes the compounds lipophilicity, in which aromatic ring makes $\pi$-overlap with the aromatic amino acid on the target receptors. Carboxylate moiety gives new compounds hydrophilicity, and forms electrostatic bonding (dipole-dipole interaction) with corresponding receptors, the hydrocarbons in chloroformate provide lipophilic interaction with corresponding receptors. These forces of interaction expected to provide the potential antimicrobial activity.

\section{MATERIALS AND METHODS}

\section{Experimental}

\section{Chemistry}

\section{Chemicals}

2-mercaptobenzothiazole 97\%, propargyl bromide, ethyl chloroformate $97 \%$, methyl chloroformate, isobutyl chloroformate, cyclic amine: 1-methylpiperazine 99\%, 2-methylpiperidine 98\%, cis-2,6dimethylpiperidine 98\%, hexamethylenediamine (azepane) 99\%, pyrrolidine 99\%, piperidine 99\% (all were from Sigma Aldrich, USA), magnesium sulphate anhydrous (Lonover, UK), potassium bromide (Scharlau, Spain), potassium carbonate anhydrous (Gainland Chemical Company, UK), potassium hydroxide (Lonover, UK), paraformaldehyde polymer (BDH chemicals Ltd Poole, England), cuprous chloride LRG (East Anglia Chemicals Hadleigh lpswich), acetonitrile 99.7\% (PanReAc Quimca SA, EU), 1,4-dioxane (FULL Time, China), chloroform (stabilized with $0.5-1 \%$ ethanol) (TEDIA, USA), dimethyl sulfoxide (DMSO) (BBC Chemicals for lab, EU), diethyl ether (Lonover, England)/(RCL Labs can, Thailand), absolute ethanol 99.9\% (Super Chem Inc, Sarasota), acetone 99\% (Scharlau, Spain).

\section{Instrumentation}

Analytical balance with a precision $0.01 \mathrm{mg}$ (Phoenix instrument, USA), hot plate with magnetic stirrer (Dragon, China), rotary evaporator 0-100Kpa/0-700 mmHg (Rocker 600, Germany), buchner funnel pump (Vacuubrand, Germany), melting point apparatus (Gallenkamp, USA), FT-IR spectrophotometer 7800 to 400 cm-1 (Evisa, Poland), DSC (Mettler Toledo, Int Co), UV-VIS (Evolution 160,USA), HPLC-UV (FinniGan Surveyor, USA), NMR 300 MHZ (Varian 300 MHZ, USA), NMR 500 MHZ (Varian 500 MHZ, USA), elemental analyzer with variation range $( \pm 4)$ (Euro Vector, Italy), balance (BoEco, Germany), autoclave machine (Rypa, Spain), incubator (EuroStar, EU), vortex mixer (Labinco, India), hot plate magnetic stirrer (Dragon, China), sterile tubes, sterile swabs (mWe, UK), micropipette (Oxford, USA).

\section{Synthesis}

\section{Synthesis of 2-(prop-2-yn-1-ylsulfanyl)-1,3-benzothiazole (AZ0)}

After the mixture of 2-MBT (5.01 g, 0.03 mole), potassium carbonate anhydrous ( $3 \mathrm{~g},>0.03 \mathrm{~mol}$ ) and $20-40 \mathrm{ml}$ acetonitrile (ACN) has been heated and stirred under reflux for $30 \mathrm{~min}$, the propargyl bromide ( 5 $\mathrm{ml}, 0.03 \mathrm{~mol}$ ) was added dropwise. The reaction mixture was heated and stirred under reflux for $2 \mathrm{~h}$. Then the mixture was filtrated and concentrated under reduced pressure to give a brown syrup. The crude product was extracted with $50 \mathrm{ml}$ chloroform and $50 \mathrm{ml}$ distilled water; chloroform layers were collected, dried over magnesium sulphate and evaporated under reduced pressure. The solid brownish crystals $\mathrm{AZO} \mathrm{C}_{10} \mathrm{H}_{7} \mathrm{NS}_{2}, 3.4 \mathrm{~g}$, $55 \%$ yield, Mp: $\left(40{ }^{\circ} \mathrm{C}\right.$ $\left.50{ }^{\circ} \mathrm{C}\right)$, retention time UV-HPLC: $(2.91 \mathrm{~min}), \mathrm{IR}\left(\mathrm{KBr} \mathrm{cm}^{-1}\right)$ : acetylenic C-H stretching (3278.39 $\left.\mathrm{cm}^{-1}\right), \mathrm{C}-\mathrm{H}$ stretching $\operatorname{Ar}\left(3060.477 \mathrm{~cm}^{-1}\right), \mathrm{C}$ $\equiv \mathrm{C}$ stretching $\left(2125.171 \mathrm{~cm}^{-1}\right), \mathrm{C}=\mathrm{C}$ stretching $\mathrm{Ar}\left(1625 \mathrm{~cm}^{-1}\right), \mathrm{C}=\mathrm{N}$ stretching thiazole $\left(1475 \mathrm{~cm}^{-1}\right), \mathrm{C}-\mathrm{N} 3{ }^{\circ}$ aromatic $\left(1388 \mathrm{~cm}^{-1}\right), \mathrm{C}-\mathrm{H}$ out of plane bending $\mathrm{Ar}$ (700-859.739 $\mathrm{cm}-1)$, S-C stretching (600-700 $\mathrm{cm}^{-1}$ ), ${ }^{1} \mathrm{H}-\mathrm{NMR}$ (DMSO-d 6 ): $\delta ; 7.85-8$ ppm (doublet, aromatic protons, $2 \mathrm{H}$ ), 7.3-7.45 ppm (triplet, aromatic protons, $2 \mathrm{H}$ ), 4.2 ppm (singlet, S-CH2-C, 2H), 3.1 ppm (singlet, acetylenic proton $\mathrm{C} \equiv \mathrm{C}-\mathrm{H}, 1 \mathrm{H})$.

Synthesis of 2-\{[4-(t-amino-1-yl) but-2-yn-1-yl] sulfanyl $\}-1,3-$ benzothiazole derivatives by Mannich reaction (AZ1-6)

A mixture of 2-(prop-2-yn-1-ylsulfanyl)-1,3-benzothiazole (AZ0) ( $2.062 \mathrm{~g}, 0.01 \mathrm{~mol})$, Para formaldehyde $(0.5 \mathrm{~g}$ in excess), cyclic amine (1-methylpiperazine, 2-methyl piperidine, 2,6-di methyl piperidine, azepane, pyrrolidine, piperidine) and a catalytic amount of cuprous chloride in 1,4-dioxane $(25 \mathrm{ml})$ was stirred at room temperature for $10 \mathrm{~min}$ then was heated and stirred under reflux at $70-75{ }^{\circ} \mathrm{C}$ for three h. Reaction mixture was filtrated and concentrated under reduced pressure to give brown syrup which was dissolved in diethyl ether, filtrated and concentrated under reduced pressure. The final products were AZ1, AZ2, AZ3, AZ4, AZ5, and AZ6.

\section{2\{[4-(4-methylpiperazine-1-yl)but-2-yn-1-yl]sulfanyl\}-1,3- benzo-thiazole (AZ1)}

The title compound AZ1 was synthesized, using a similar procedure to that described for the preparation of 2-\{[4-(t-amino-1-yl)but-2yn-1-yl] sulfanyl $\}$-1,3-benzothiazole derivatives by Mannich reaction (AZ1-6) in $1.5 \mathrm{~g}$, yield $63.03 \%$, Mp: $\left(40^{\circ} \mathrm{C}-50{ }^{\circ} \mathrm{C}\right)$, retention time UVHPLC: $(2.2 \mathrm{~min})$, FT-IR $\left(\mathrm{KBr} \mathrm{cm}^{-1}\right)$ : C-H stretching aromatic $\left(3060.477 \mathrm{~cm}^{-1}, 2933.992 \mathrm{~cm}^{-1}\right), \mathrm{C}-\mathrm{H}$ stretching with tertiary amine $\left(2776.992 \mathrm{~cm}^{-1}\right), \mathrm{C}=\mathrm{C}$ stretching aromatic $\left(1656.553 \mathrm{~cm}^{-1}\right), \mathrm{C}=\mathrm{N}$ stretching thiazole $\left(1600 \mathrm{~cm}^{-1}\right), \quad \mathrm{C}-\mathrm{N}$ stretching 3 。 aromatic(1417.427 $\left.\mathrm{cm}^{-1}\right), \mathrm{C}-\mathrm{N}$ stretching in cyclic amine $(1276.645$ $\left.\mathrm{cm}^{-1}\right), \mathrm{C}-\mathrm{H}$ out of plane bending aromatic $\left(727.032 \mathrm{~cm}^{-1}, 809.956 \mathrm{~cm}^{-}\right.$ $\left.{ }^{1}\right)$, C-S stretching (534.186-630 $\mathrm{cm}^{-1}$ ), ${ }^{1} \mathrm{H}-\mathrm{NMR}$ (DMSO $\mathrm{d}_{6}$ ): $\delta ; 2.05$ ppm (singlet, cyclic amine proton A, 3H), 2.11 ppm (triplet, cyclic amine proton $\mathrm{B}, 4 \mathrm{H}), 2.32 \mathrm{ppm}$ (triplet, cyclic amine protons $\mathrm{C}, 4 \mathrm{H}$ ), 3.18 ppm (singlet, $\mathrm{C}-\mathrm{CH}_{2}-\mathrm{N}, 2 \mathrm{H}$ ), $4.2 \mathrm{ppm}$ (singlet, $\mathrm{S}-\mathrm{CH}_{2}-\mathrm{C}, 2 \mathrm{H}$ ), 7.35 ppm (triplet, aromatic protons type A, 2H), $7.45 \mathrm{ppm}$ (triplet, aromatic protons type $\mathrm{B}, 2 \mathrm{H}$ ), $7.85 \mathrm{ppm}$ (doublet, aromatic protons type $\mathrm{C}, 1 \mathrm{H}$ ), $8 \mathrm{ppm}$ (doublet, aromatic protons type $\mathrm{D}, 1 \mathrm{H})$. [13]CNMR (DMSO d $\mathrm{d}_{6}$ ): $\delta ; 22.1289$ ppm (cyclic amine $\mathrm{CH}_{3}$ carbon), 46.1170 ppm 46.704 ppm (cyclic amine carbons), $55.0402 \mathrm{ppm}\left(\mathrm{CH}_{2} \mathrm{~N}\right)$, 97.954, 80.34 (C $\equiv \mathrm{C}), 51.536\left(\mathrm{SCH}_{2}\right), 165.50,153.074,135.38$, 122.3883, 121.8148, 126.8, 125.0743 (2-MPT carbons). Elemental analysis: calculated for $\mathrm{C}_{16} \mathrm{H}_{19} \mathrm{~N}_{3} \mathrm{~S}_{2} ; \mathrm{C} 60.53 \%, \mathrm{H} 6.03 \%, \mathrm{~N} 13.24 \%$. Found: C $60.219 \%$, H 6.35\%, N13.702\%.

\section{2-\{[4-(2-methylpiperidine-1-yl)but-2-yn-1-yl]sulfanyl\}-1,3- benzothiazole (AZ2)}

The title compound AZ2 was synthesized, using a similar procedure to that described for the preparation of $2-\{[4$-(t-amino-1-yl)but-2yn-1-yl] sulfanyl\}-1,3-benzothiazole derivatives by Mannich reaction (AZ1-6) in 1.2 g, yield $42.47 \%$, Mp: $\left(40-50^{\circ} \mathrm{C}\right)$, UV-HPLC retention time (2.1 min), FT-IR ( $\left.\mathrm{KBr} \mathrm{cm}^{-1}\right)$ : C-H stretching aromatic (3060.477 $\left.\mathrm{cm}^{-1}, 2923.556 \mathrm{~cm}^{-1}\right), \mathrm{C}-\mathrm{H}$ stretching tertiary amine $\left(2788.563 \mathrm{~cm}^{-1}\right.$, 2744.208-1), C=C stretching aromatic (1673.909 $\left.\mathrm{cm}^{-1}\right), \quad \mathrm{C}=\mathrm{N}$ stretching thiazole $\left(1434.779 \mathrm{~cm}^{-1}\right), \mathrm{C}-\mathrm{N}$ stretching $3^{\circ}$ aromatic $\left(1307.283 \mathrm{~cm}^{-1}\right), \mathrm{C}-\mathrm{N}$ stretching tertiary cyclic amine $(1228.433 \mathrm{~cm}$ 1), C-H out of plane bending aromatic $(879.381 \mathrm{~cm}-1), \mathrm{C}-\mathrm{S}$ stretching (628.68 $\mathrm{cm}^{-1}, 730.889 \mathrm{~cm}^{-1}$ ). ${ }^{1} \mathrm{H}-\mathrm{NMR}$ (DMSO d6): $\delta$; cyclic amine protons: $0.75 \mathrm{ppm}$ (doublet, type A, $3 \mathrm{H}$ ) $0.95 \mathrm{ppm}$ (sextet, type $\mathrm{B}, 1 \mathrm{H}), 1.2 \mathrm{ppm}$ (quintet, type $\mathrm{C}, 2 \mathrm{H}$ ), $1.4 \mathrm{ppm}$ (quartet, type $\mathrm{D}, 2 \mathrm{H}$ ), 1.9 ppm (triplet, type E, 2H), $2.1 \mathrm{ppm}$ (quintet, type $\mathrm{F}, 2 \mathrm{H}$ ), $3.3 \mathrm{ppm}$ (singlet, $\mathrm{C}-\mathrm{CH}_{2}-\mathrm{N}, 2 \mathrm{H}$ ), $4.3 \mathrm{ppm}$ (singlet, $\mathrm{S}-\mathrm{CH}_{2}-\mathrm{C}, 2 \mathrm{H}$ ), four aromatic protons: $7.35 \mathrm{ppm}$ (triplet, type A, $2 \mathrm{H}$ ), $7.45 \mathrm{ppm}$ (triplet, type B, $2 \mathrm{H}), 7.85 \mathrm{ppm}$ (doublet, type C, 1H), 8 ppm (doublet, type $\mathrm{D}, 1 \mathrm{H}$ ).

\section{2-\{[4-(2,6-dimethylpiperidin-1-yl)but-2-yn-1-yl]sulfanyl\}-1,3- benzothiazole (AZ3)}

The title compound AZ3 was synthesized, using a similar procedure to that described for the preparation of $2-\{[4-(t-a m i n o-1-y l) b u t-2$ yn-1-yl] sulfanyl\}-1,3-benzothiazole derivatives by Mannich reaction (AZ1-6) in 2.5 g, yield $76.22 \%$, Mp: $\left(42-55^{\circ} \mathrm{C}\right)$, UV-HPLC retention time $(2.167 \mathrm{~min}), \mathrm{FT}-\mathrm{IR}\left(\mathrm{KBr} \mathrm{cm} \mathrm{cm}^{-1}\right): \mathrm{C}-\mathrm{H}$ stretching aromatic (3080.477 $\left.\mathrm{cm}^{-1}, 2923.556 \mathrm{~cm}^{-1}\right), \mathrm{C}-\mathrm{H}$ stretching tertiary amine $\left(2850.274 \mathrm{~cm}^{-1}, 2788.563 \mathrm{~cm}^{-1}, \mathrm{C}=\mathrm{C}\right.$ stretching aromatic $(1673.909$ 
$\left.\mathrm{cm}^{-1}\right), \mathrm{C}=\mathrm{N}$ stretching thiazole $\left(1590.985 \mathrm{~cm}^{-1}, 1432.851 \mathrm{~cm}^{-1}\right), \mathrm{C}-\mathrm{N}$ stretching $3^{\circ}$ aromatic $\left(1373.168 \mathrm{~cm}^{-1}\right), \mathrm{C}-\mathrm{N}$ stretching tertiary cyclic amine (12226.433 $\left.\mathrm{cm}^{-1}\right), \mathrm{C}-\mathrm{H}$ out of plane bending aromatic (865.882 $\left.\mathrm{cm}^{-1}\right)$, C-S stretching $\left(717.39 \mathrm{~cm}^{-1}, 624.823 \mathrm{~cm}^{-1}\right),{ }^{1} \mathrm{H}-\mathrm{NMR}$ (DMSO $\mathrm{d}_{6}$ ): $\delta$; four types of cyclic amine protons: $0.75 \mathrm{ppm}$ (doublet, type A, 6H), 0.95 ppm (sextet, type B, 2H) 1.2-1.35 ppm (quartet, type $\mathrm{C}, 4 \mathrm{H}$ ), $2 \mathrm{ppm}$ (quintet, type $\mathrm{D}, 2 \mathrm{H}$ ), $3.4 \mathrm{ppm}$ (singlet, $\mathrm{C}-\mathrm{CH}_{2}-\mathrm{N}$, $2 \mathrm{H}$ ), $4.2 \mathrm{ppm}$ (singlet, $\mathrm{S}-\mathrm{CH}_{2} \mathrm{C}, 2 \mathrm{H}$ ), four types of aromatic protons: $7.35 \mathrm{ppm}$ (triplet, type A, 2H), $7.45 \mathrm{ppm}$ (triplet, type $\mathrm{B}, 2 \mathrm{H}$ ), 7.85 ppm (doublet, type C, 1H), 8 ppm (doublet, type $\mathrm{D}, 1 \mathrm{H}$ ).

\section{2-\{[4-(azepane-1-yl) but-2-yn-1-yl] sulfanyl\}-1,3-benzothiazole (AZ4)}

The title compound AZ4 was synthesized, using a similar procedure to that described for the preparation of 2-\{[4-(t-amino-1-yl)but-2-yn-1yl] sulfanyl\}-1,3-benzothiazole derivatives by Mannich reaction (AZ16) in $2.74 \mathrm{~g}$, yield $100 \%$, Mp: $\left(50-60{ }^{\circ} \mathrm{C}\right)$, UV-HPLC retention time (2.117 min), FT-IR $\left(\mathrm{KBr} \mathrm{cm}^{-1}\right)$ : C-H stretching aromatic $\left(3056.62 \mathrm{~cm}^{-1}\right.$, $\left.2919.699 \mathrm{~cm}^{-1}\right)$, C-H stretching tertiary amine $\left(2846.417 \mathrm{~cm}^{-1}\right.$, $\left.2809.776 \mathrm{~cm}^{-1}\right), C=C$ stretching aromatic $\left(1677.766 \mathrm{~cm}^{-1}\right), C=N$ stretching thiazole $\left(1583.271 \mathrm{~cm}^{-1}, 1417.423 \mathrm{~cm}^{-1}\right), \mathrm{C}-\mathrm{N}$ stretching $3^{\circ}$ aromatic $\left(1321 \mathrm{~cm}^{-1}\right), \mathrm{C}-\mathrm{N}$ stretching(new bond) tertiary cyclic amine (1232.29 $\left.\mathrm{cm}^{-1}\right), \mathrm{C}-\mathrm{H}$ out of plane bending aromatic $\left(879.381 \mathrm{~cm}^{-1}\right), \mathrm{C}-\mathrm{S}$ stretching (721.247 $\left.\mathrm{cm}^{-1}, 628.68 \mathrm{~cm}^{1}\right),{ }^{1} \mathrm{H}-\mathrm{NMR}$ (DMSO $\mathrm{d}_{6}$ ): $\delta$; two types of cyclic amine protons: $1.35 \mathrm{ppm}$ (quartet, type A, 10H), 2.35 ppm (triplet, type B, 4H), $3.2 \mathrm{ppm}$ (singlet, $\mathrm{C}-\mathrm{CH}_{2}-\mathrm{N}, 2 \mathrm{H}$ ), $4.2 \mathrm{ppm}$ (singlet, $\mathrm{S}-\mathrm{CH}_{2}-\mathrm{C}, 2 \mathrm{H}$ ), four types of aromatic protons: $7.35 \mathrm{ppm}$ (triplet, type A, 2H), $7.45 \mathrm{ppm}$ (triplet, type B, 2H), $7.85 \mathrm{ppm}$ (doublet, type $\mathrm{C}, 1 \mathrm{H}$ ), 8 ppm (doublet, type $\mathrm{D}, 1 \mathrm{H}$ ).

\section{2-\{[4-(pyrrolidin-1-yl) but-2-yn-1-yl] sulfanyl\}-1,3-benzothiazole (AZ5)}

The title compound AZ5 was synthesized, using a similar procedure to that described for the preparation of 2-\{[4-(t-amino-1-yl)but-2yn-1-yl] sulfanyl $\}$-1,3-benzothiazole derivatives by Mannich reaction (AZ1-6) in $1.99 \mathrm{~g}$, yield $69.097 \%$, Mp: $\left(25-38{ }^{\circ} \mathrm{C}\right)$, UV-HPLC retention time (1.99 min), FT-IR $\left(\mathrm{KBr} \mathrm{cm}^{-1}\right)$ : C-H stretching aromatic $\left(3048.906 \mathrm{~cm}^{-1}, 2958.265 \mathrm{~cm}^{-1}\right), \mathrm{C}-\mathrm{H}$ stretching tertiary amine (2958.274 $\left.\mathrm{cm}^{-1}, 2796.277 \mathrm{~cm}^{-1}\right), \mathrm{C}=\mathrm{C}$ stretching aromatic (1592.92 $\left.\mathrm{cm}^{-1}\right), \mathrm{C}=\mathrm{N}$ stretching thiazole $\left(1415.495 \mathrm{~cm}^{-1}\right), \mathrm{C}-\mathrm{N}$ stretching $3^{\circ}$ aromatic $\left(1307.501 \mathrm{~cm}^{-1}\right)$, C-N stretching tertiary cyclic amine (new) $\left(1241.933 \mathrm{~cm}^{-1}\right), \mathrm{C}-\mathrm{H}$ out of plane bending aromatic $\left(865.882 \mathrm{~cm}^{-1}\right)$, C-S stretching (721.247 $\mathrm{cm}^{-1}$ ), ${ }^{1} \mathrm{H}-\mathrm{NMR}$ (DMSO $\mathrm{d}_{6}$ ): $\delta$; two types of cyclic amine protons: $1.5 \mathrm{ppm}$ (quartet, type $\mathrm{A}, 4 \mathrm{H}$ ), $2.3 \mathrm{ppm}$ (triplet, type B, $4 \mathrm{H}$ ), $3.3 \mathrm{ppm}$ (singlet, $\mathrm{C}_{-} \mathrm{CH}_{2}-\mathrm{N}, 2 \mathrm{H}$ ), $4.2 \mathrm{ppm}$ (singlet, $\mathrm{S}^{-\mathrm{CH}_{2}-}$ $\mathrm{N}, 2 \mathrm{H}$ ), four types of aromatic protons: $7.35 \mathrm{ppm}$ (triplet, type $\mathrm{A}, 2 \mathrm{H}$ ), $7.45 \mathrm{ppm}$ (triplet, type $\mathrm{B}, 2 \mathrm{H}$ ), $7.85 \mathrm{ppm}$ (doublet, type $\mathrm{C}, 1 \mathrm{H}$ ), $8 \mathrm{ppm}$ (doublet, type D, 1H). ${ }^{13} \mathrm{C}$-NMR (DMSO $\mathrm{d}_{6}$ ): $\delta$; $22.1979 \mathrm{ppm}, 23.6$ ppm, 23.6002, 23.6757 (cyclic amine carbons), $42.6\left(\mathrm{CH}_{2} \mathrm{~N}\right), 79.8806$ ppm, 80.182 ppm $(\mathrm{C} \equiv \mathrm{C}), 51.811 \mathrm{ppm}\left(\mathrm{SCH}_{2}\right), 165.47 \mathrm{ppm}, 153.104$ ppm, 135.3731 ppm, 121.7915 ppm, 122.2931 ppm, 125.09 ppm, $126.8803 \mathrm{ppm}$ (2-MBT carbons), Elemental analysis; calculated for $\mathrm{C}_{15} \mathrm{H}_{16} \mathrm{~N}_{2} \mathrm{~S}_{2}$, C $62.40 \%, \mathrm{H}$ 8.50, N 9.71\%. Found: C $62.134 \%, \mathrm{H}$ $6.176 \%$, N $10.054 \%$.

\section{2-\{[4-(piperidin-1-yl) but-2-yn-1-yl] sulfanyl\}-1,3-benzothiazole (AZ6)}

The title compound AZ6 was synthesized, using a similar procedure to that described for the preparation of $2-\{[4-(t-a m i n o-1-y l) b u t-2-$ yn-1-yl] sulfanyl\}-1,3-benzothiazole derivatives by Mannich reaction (AZ1-6) in $1.99 \mathrm{~g}$, yield $65.894 \%$, Mp: $\left(70-79^{\circ} \mathrm{C}\right)$, UV-HPLC retention time $(2.07 \mathrm{~min})$, FT-IR $(\mathrm{KBr} \mathrm{cm}-1)$ : $\mathrm{C}-\mathrm{H}$ stretching aromatic (3043.121 $\mathrm{cm}^{-1}, 2923.556 \mathrm{~cm}^{-1}, \mathrm{C}-\mathrm{H}$ stretching tertiary amine $\left(2848.274 \mathrm{~cm}^{-1}, 2788.563 \mathrm{~cm}^{-1}, \mathrm{C}=\mathrm{C}\right.$ stretching aromatic $(1668.124$ $\left.\mathrm{cm}^{-1}\right), \mathrm{C}=\mathrm{N}$ stretching thiazole $\left(1587.128 \mathrm{~cm}^{-1}, 1455.993 \mathrm{~cm}^{-1}\right), \mathrm{C}-\mathrm{N}$ stretching $3^{\circ}$ aromatic $\left(1315.214 \mathrm{~cm}^{-1}\right), \mathrm{C}-\mathrm{N}$ stretching new tertiary cyclic amine bond $\left(1232.29 \mathrm{~cm}^{-1}\right), \mathrm{C}-\mathrm{H}$ out of plane bending aromatic $\left(879.361 \mathrm{~cm}^{-1}\right), \mathrm{C}-\mathrm{S}$ stretching $\left(636.394 \mathrm{~cm}^{-1}, 734.746 \mathrm{~cm}^{-1}\right),{ }^{1} \mathrm{H}-$ NMR (DMSO $\mathrm{d}_{6}$ ): $\delta$; two types of cyclic amines protons: $1.3 \mathrm{ppm}$ (quintet, type A, 6H), $2.2 \mathrm{ppm}$ (triplet, type $\mathrm{B}, 4 \mathrm{H}$ ), $3.15 \mathrm{ppm}$ (singlet,

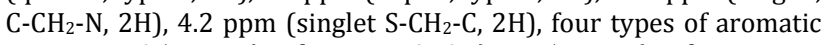
protons: $7.35 \mathrm{ppm}$ (triplet, type $\mathrm{A}, 2 \mathrm{H}$ ), $7.45 \mathrm{ppm}$ (triplet, type $\mathrm{B}$, $2 \mathrm{H}$ ), $7.85 \mathrm{ppm}$ (doublet, type $\mathrm{C}, 1 \mathrm{H}$ ), $8 \mathrm{ppm}$ (doublet, type $\mathrm{D}, 1 \mathrm{H}$ ), [13]C-NMR (DMSO d $\mathrm{d}_{6}$ ): $22.2392 \mathrm{ppm}, 23.7826 \mathrm{ppm}, 25.7792 \mathrm{ppm}$ (cyclic amine carbons), $47.4668 \mathrm{ppm}\left(\mathrm{CH}_{2} \mathrm{~N}\right), 79.9823 \mathrm{ppm}, 80.3944$ ppm (C三C), $52.6517 \mathrm{ppm}\left(\mathrm{SCH}_{2}\right), 165.4149 \mathrm{ppm}, 153.1251 \mathrm{ppm}$, 135.4022 ppm, 121.8065 ppm, 122.27676 ppm, 125.1017 ppm, $126.8625 \mathrm{ppm}$ (2-MBT carbons), Elemental analysis; calculated for $\mathrm{C}_{16} \mathrm{H}_{18} \mathrm{~N}_{2} \mathrm{~S} 2$, C $62.7 \%$, H 5.8\%, N 10\%. Found: C 63.785\%, H 6.368\%, N $9.332 \%$.

\section{Synthesis of S-1,3-benzothiazol-2-yl-0-t carbonothioate (AZ7-9)}

After the mixture of 2-MBT ( 5 g, 0.03 mole), Potassium Hydroxide $(1.6 \mathrm{~g}, 0.03)$, anhydrous potassium carbonate $(3 \mathrm{~g}, 0.03)$ in $20-30 \mathrm{ml}$ absolute ethanol has been heated and stirred under reflux for 30-45 min, it was cooled to room temperature. The appropriate (methyl, ethyl, Isobutyl) chloroformate (0.03 mol) was added dropwise with continuous stirring then it was heated to $30-35{ }^{\circ} \mathrm{C}$ for $1-2 \mathrm{~h}$. After the mixture has been cooled to room temperature, it was poured into $100 \mathrm{ml}$ iced water, the precipitated product was filtered by buchner funnel and washed with a little amount of cold distilled water. Finally, the white crystals formed were recrystallized from ethanolwater, the final products AZ7, AZ8, and AZ9.

\section{S-1,3-benzothiazol-yl-0-ethyl carbonothioate (AZ7)}

The title compound AZ7 was synthesized, using a similar procedure to that described for the preparation of synthesis of S-1,3benzothiazol-2-yl-0-t carbonothioate derivatives by addition chloroformate (AZ7-9) in 6 g, Yield 83.57\%, Mp: (60-65 ${ }^{\circ} \mathrm{C}$ ), UVHPLC retention time (3.485 min), FT-IR $\left(\mathrm{KBr} \mathrm{cm}^{-1}\right)$ : C-H stretching aromatic $\left(2994.909 \mathrm{~cm}^{-1}, \mathrm{C}=0\right.$ stretching ester formate $(1727.906$ $\left.\mathrm{cm}^{-1}\right), \mathrm{C}=\mathrm{C}$ stretching aromatic $\left(1600 \mathrm{~cm}^{-1}\right), \mathrm{C}=\mathrm{N}$ stretching thiazole $\left(1458.993 \mathrm{~cm}^{-1}, 1411.638 \mathrm{~cm}^{-1}\right), \mathrm{C}-\mathrm{N} 3^{\circ}$ aromatic $\left(1369.212 \mathrm{~cm}^{-1}\right), \mathrm{C}-$ $\mathrm{O}$ stretching ester $\left(1153.233 \mathrm{~cm}^{-1}\right), \mathrm{C}-\mathrm{H}$ bending out of plane aromatic $\left(849.525 \mathrm{~cm}^{-1}\right)$, C-S stretching $\left(655.979 \mathrm{~cm}^{-1}, 725.104 \mathrm{~cm}^{-}\right.$ 1), ${ }^{1} \mathrm{H}-\mathrm{NMR}$ (DMSO d6): $\delta ; 1.3 \mathrm{ppm}$ (triplet, $\mathrm{CH}_{3}$ Protons, $3 \mathrm{H}$ ), $4.2 \mathrm{ppm}$ (quartet, $\mathrm{CH}_{2}$ protons, $2 \mathrm{H}$ ), 7.5 ppm-8.3 ppm (aromatic protons).

\section{S-1,3-benzothizol-2-yl-0-methyl carbonothioate (AZ8)}

The title compound AZ8 was synthesized, using a similar procedure to that described for the preparation of synthesis of $\mathrm{S}-1,3$ benzothiazol-2-yl-0-t carbonothioate derivatives by addition chloroformate (AZ7-9) in $5.4 \mathrm{~g}$, yield $79.917 \%$, UV-HPLC retention time $(2.893 \mathrm{~min})$, FT-IR $\left(\mathrm{KBr} \mathrm{cm} \mathrm{cm}^{-1}\right): \mathrm{C}-\mathrm{H}$ stretching aromatic (2942.909 $\mathrm{cm}^{-1}, \mathrm{C}=0$ stretching ester formate $\left(1718.906 \mathrm{~cm}^{-1}\right), \mathrm{C}=\mathrm{C}$ stretching aromatic $\left(1967 \mathrm{~cm}^{-1}\right), \mathrm{C}=\mathrm{N}$ stretching thiazole $(1411.638$ $\left.\mathrm{cm}^{-1}\right), \quad \mathrm{C}-\mathrm{N} 3{ }^{\circ}$ aromatic $\left(1307.212 \mathrm{~cm}^{-1}\right)$, C-O stretching ester $\left(1132.233 \mathrm{~cm}^{-1}\right), \mathrm{C}-\mathrm{H}$ bending out of plane aromatic $\left(817.525 \mathrm{~cm}^{-1}\right)$, C-S stretching ( $\left.655.979 \mathrm{~cm}^{-1}, 755.104 \mathrm{~cm}^{-1}\right),{ }^{1} \mathrm{H}-\mathrm{NMR}$ (DMSO d6): $\delta ; 4$ ppm (singlet, $\mathrm{CH}_{3}$ protons, $3 \mathrm{H}$ ), 7.5 ppm-8.15 ppm (aromatic protons), elemental analysis; calculated for $\mathrm{C}_{9} \mathrm{H}_{7} \mathrm{NO}_{2} \mathrm{~S}_{2}, \mathrm{C} 47.96 \%, \mathrm{H}$ $3.13 \%$, N 6.22\%. Found: C 48.131\%, H 2.801\%, N 6.094.

\section{S-1,3-benzothiazol-2-yl-0-(2-methyl propyl) carbonothioate (AZ9)}

The title compound AZ9 was synthesized, using a similar procedure to that described for the preparation of synthesis of S-1,3benzothiazol-2-yl-O-t-carbonothioate derivatives by addition chloroformate (AZ7-9) in $5.8 \mathrm{~g}$, yield $72 \%$, UV-HPLC retention time (5.547 min), FT-IR $\left(\mathrm{KBr} \mathrm{cm}^{-1}\right): \mathrm{C}-\mathrm{H}$ stretching aromatic (3064.334 $\left.\mathrm{cm}^{-1}, 2942.909 \mathrm{~cm}^{-1}\right), \mathrm{C}=0$ stretching ester/formate $\left(1772.121 \mathrm{~cm}^{-1}\right)$, $\mathrm{C}=\mathrm{C}$ stretching aromatic $\left(1625 \mathrm{~cm}^{-1}\right), \mathrm{C}=\mathrm{N}$ stretching thiazole (1463.706 $\left.\mathrm{cm}^{-1}\right), \mathrm{C}-\mathrm{N} 3^{\circ}$ aromatic $\left(1307.212 \mathrm{~cm}^{-1}\right), \mathrm{C}-0$ stretching ester $\left(1159.009 \mathrm{~cm}^{-1}\right), \mathrm{C}-\mathrm{H}$ bending out of plane aromatic $\left(804 \mathrm{~cm}^{-1}\right.$, $\left.873.596 \mathrm{~cm}^{-1}\right)$, C-S stretching $\left(655.979 \mathrm{~cm}^{-1}, 755.104 \mathrm{~cm}^{-1}\right),{ }^{1} \mathrm{H}-\mathrm{NMR}$ (DMSO $\mathrm{d}_{6}$ ): $\delta ; 0.85 \mathrm{ppm}\left(\mathrm{CH}_{3}\right.$ protons, doublet, $\left.6 \mathrm{H}\right), 1.9 \mathrm{ppm}(\mathrm{CH}$ proton, 9 signals, $1 \mathrm{H}), 4.15 \mathrm{ppm}\left(\mathrm{CH}_{2}\right.$ proton, doublet, $\left.2 \mathrm{H}\right), 7.5-8.15$ ppm (aromatic protons), [13]C-NMR (DMSO $\mathrm{d}_{6}$ ): 18.9323, 19.0954, $19.1977 \mathrm{ppm}\left(\mathrm{CH}_{3}\right), 27.7733 \mathrm{ppm}(\mathrm{CH}), 75.4644 \mathrm{ppm}\left(\mathrm{CH}_{2}\right)$, $157.6041 \mathrm{ppm}$ (SCOOR), $165.6388 \mathrm{ppm}, 152.6042 \mathrm{ppm}$ ), 136.488 ppm, 126.332 ppm, 127.1825 ppm, 122.5017 ppm 123.0872 ppm (2MBT carbons).

\section{Culture media}

Mueller-Hinton agar (MHA) (Mastgrp Ltd, UK), Muller Hinton broth (MHB) (Mastgrp Ltd, UK), sabourauds dextrose agar (SDA) (Mastgrp Ltd, UK), sabourauds dextrose broth (SDB) (Himedia, India). 


\section{Microorganism}

Staphylococcus aureus (S. aureus ATCC 6538), Bacillus subtilis (B. Subtilis ATCC 6633), Pseudomonas aeruginosa (P. aeruginosa ATCC 9027), Escherichia coli (E. coli ATCC 8739), Candida albicans (C. albicans ATCC 10231). All these pure cultures of bacterial strains were kindly obtained from Dar Al Dawa (Na'ur, Jordan).

\section{Antimicrobial activity testing}

All the newly synthesized compounds 2-\{[4-(t-amino-1-yl)but-2-yn-1-yl] sulfanyl $\}$-1,3-benzothiazole derivatives (AZ1-6) and S-1,3-benzothiazol2-yl-0-t carbonothioate derivatives (AZ7-9) were tested in vitro for antimicrobial activity against two Gram-negative bacteria namely E. coli (ATCC 8739) and P. aeruginosa (ATCC 9027), two Gram-positive bacteria namely S. aureus (ATCC 6538p) and B. subtilis (ATCC 6633), and one fungi namely C. albicans (ATCC 10231). The first method used was agar diffusion method measuring the zone of inhibition diameter, the compound solutions (AZ1-9) have been prepared in different concentrations for each compound ( $500 \mu \mathrm{g} / \mathrm{ml}, 250 \mu \mathrm{g} / \mathrm{ml}, 125 \mu \mathrm{g} / \mathrm{ml}$, $62.5 \mu \mathrm{g} / \mathrm{ml}$ ). After overnight culturing of bacteria in MHB and $2 \mathrm{~d}$ culturing of fungi in SDB at $37^{\circ} \mathrm{C}, 0.1 \mathrm{ml}$ of $1 \mathrm{X} 10^{6} \mathrm{CFU} / \mathrm{ml}$ of bacterial or fungal broth suspension was inoculated over the surface of MHA plates for bacteria or SDA plates for fungi. Then wells were cut into the media. The wells were filled serially with $0.2 \mathrm{ml}$ compounds' solutions. Plates were incubated at $37^{\circ} \mathrm{C}$ for $24 \mathrm{~h}$ for bacteria and $48 \mathrm{~h}$ for fungi. The zone of inhibition diameters formed around the wells was measured, then they were compared to the zone diameter formed around the positive controls (ciprofloxacin disk $5 \mu \mathrm{g}$, fluconazole $500 \mu \mathrm{g} / \mathrm{ml}$ in DMSO). Negative control (DMSO) was also included. Agar diffusion method was designed to allow for statistical analysis and were performed in triplicates. Experimental data presented in this study represent the mean $\pm S D$ of those triplicate data sets. The second method used was broth dilution method to determine the minimal inhibitory concentration (MIC) of the synthesised compounds. The synthesised compounds were diluted out in a series of twofold dilutions in broth using MHB for bacteria and SDB for fungi to give concentrations ranging from $500 \mathrm{mcg} / \mathrm{ml}$ to $7.81 \mathrm{mcg} / \mathrm{ml}$, the final volume of each dilution in the test tube was $5 \mathrm{ml} .0 .1 \mathrm{ml}$ of the overnight bacterial culture and $0.2 \mathrm{ml}$ of 2-day fungal culture were added to each tube, positive control tube containing $5 \mathrm{ml}$ of sterile $\mathrm{MHB} / \mathrm{SDB}$ and $0.1 \mathrm{ml}$ of bacterial culture $/ 0.2 \mathrm{ml}$ of fungal culture was included. Negative control tube contained $5 \mathrm{ml}$ of the tested compound dilution in sterile MHB/SDB was added. The tubes were incubated at $37^{\circ} \mathrm{C}$ for $24 \mathrm{~h}$ and $48 \mathrm{~h}$ for bacteria and fungi respectively. The MIC was determined by comparison the turbidity of each concentration tube with the positive control tube; MIC tube is the lowest concentration of the compound in which no turbidity was observed. Minimum bacteriocidial/fungicidal concentration (MBC/MFC) is the lowest concentration of antibiotic required to kill specific microorganism [11]. To determine the minimum bacteriocidial/ fungicidal concentration (MBC/MFC), the MIC tube and the tubes with dilutions preceded were cultured onto MHA/SDA plates, then the plates were incubated at $37^{\circ} \mathrm{C}$ for $24 \mathrm{~h}$ and $48 \mathrm{~h}$ for bacteria and fungi respectively. The lowest concentration tube that gave no growth was the MBC/MFC; broth dilution test was performed in triplicates.

\section{Statistical analysis}

Statistical analysis was carried using statistical packages for social science software (SPSS) for student's t-test. Values are expressed as mean $\pm S D$.

\section{RESULTS}

\section{Antimicrobial activity}

The newly synthesised compounds AZ1-9 showed activity against all types of the tested microorganisms (table 1,2), after $24 \mathrm{~h}$ incubation at $37^{\circ} \mathrm{C}$ for bacteria and after $48 \mathrm{~h}$ incubation at $37^{\circ} \mathrm{C}$ for fungi, the zone of inhibition diameter and minimum inhibitory concentration (MIC) were measured. 2-\{[4-(4-pyrrolidin-1-yl)but-2-yn-1-yl] Sulfanyl\}-1,3-benzothiazole (AZ6) demonstrated the highest antimicrobial activity against $P$. Aeruginosa, with the lowest MIC value $62.5 \mu \mathrm{g} / \mathrm{ml}$ and zone of inhibition diameter of $20 \pm 8 \mathrm{~mm}, 16 \pm 7$ $\mathrm{mm}, 16 \pm 8 \mathrm{~mm}$ and $16 \pm 8 \mathrm{~mm}$ for $500 \mu \mathrm{g} / \mathrm{ml}, 250 \mu \mathrm{g} / \mathrm{ml}, 125 \mu \mathrm{g} / \mathrm{ml}$ and $62.5 \mu \mathrm{g} / \mathrm{ml}$ respectively. Compound 2-\{[4-(4-azepan-1-yl)but-2yn-1-yl]sulfanyl\}-1,3-benzothiazole (AZ5) had the best antimicrobial activity against E. coli, with the lowest MIC value $125 \mu \mathrm{g} / \mathrm{ml}$ and zone of inhibition diameter of $11 \pm 1 \mathrm{~mm}$ and $10 \pm 0 \mathrm{~mm}$ for concentrations $500 \mu \mathrm{g} / \mathrm{ml}$ and $250 \mu \mathrm{g} / \mathrm{ml}$ respectively. $2-\{[4-(2,6-$ dimethyl-piperidin-1-yl)but-2-yn-1-yl] sulfanyl\}-1,3-benzothiazole (AZ4) had the best antimicrobial activity against $S$. aureus, with the lowest MIC value $125 \mu \mathrm{g} / \mathrm{ml}$ and zone of inhibition diameter of $20 \pm 2$ $\mathrm{mm}, 10.6 \pm 4 \mathrm{~mm}$ for concentrations $500 \mu \mathrm{g} / \mathrm{ml}$ and $250 \mu \mathrm{g} / \mathrm{ml}$ respectively. Compounds 2-\{[4-(2-methylpiperidin-1-yl)but-2-yn-1yl]sulfanyl $\}$-1,3-benzothiazole (AZ2), 2-\{[4-(2,6-dimethylpiperidin-1yl)but-2-yn-1-yl]sulfanyl\}-1,3-benzothiazole (AZ3), 2-\{[4-(2,6dimethylpiperidin-1-yl)but-2-yn-1-yl]sulfanyl $\}-1,3$-benzothiazole (AZ4) and 2-\{[4-(4-azepan-1-yl)but-2-yn-1-yl] sulfanyl $\}-1,3$-benzothiazole (AZ5) had the best antimicrobial activity against $B$. subtilis, with the lowest MIC value $62.5 \mu \mathrm{g} / \mathrm{ml}$, all these compounds do not have zone of inhibition against B. subtilis. All compounds (AZ1-9) except AZ8 had the same MBC value $(250 \mu \mathrm{g} / \mathrm{ml})$ against $P$. aeruginosa, while compounds AZ4 and AZ5 had the lowest MBC value $250 \mu \mathrm{g} / \mathrm{ml}$ against E. coli and S. aureus. Compounds AZ2, AZ3, AZ4 and AZ5 had the lowest MBC value $125 \mu \mathrm{g} / \mathrm{ml}$ against $B$. subtilis. Compound AZ9 had the lowest MIC value $31.25 \mu \mathrm{g} / \mathrm{ml}$ against $C$. albicans, with zone of inhibition diameter of $19 \pm 1 \mathrm{~mm}, 22 \pm 8 \mathrm{~mm}, 17.0 \pm 5 \mathrm{~mm}$ and $15.0 \pm 5$ $\mathrm{mm}$ for concentrations $500 \mu \mathrm{g} / \mathrm{ml}, 250 \mu \mathrm{g} / \mathrm{ml}, 125 \mu \mathrm{g} / \mathrm{ml}$ and 62.5 $\mu \mathrm{g} / \mathrm{ml}$ respectively; Accordingly, it had the lowest MFC value 62.5 $\mu \mathrm{g} / \mathrm{ml}$ against $C$. albicans. Compounds (AZ1-9) had higher MIC/MFC values in comparison to the positive control (fluconazole). The antimicrobial results of the newly synthesized compounds AZ1-9 showed a broad spectrum of antibacterial and antifungal activity.

Table 1: The zone of inhibition diameter (in mm) of compounds (AZ1-9) $500 \mu \mathrm{g} / \mathrm{ml}$ concentration

\begin{tabular}{|c|c|c|c|c|c|}
\hline Compound & P. aeruginosa & E. coli & S. aureus & B. subtilis & C. albicans \\
\hline Concentration & 500 & 500 & 500 & 500 & 500 \\
\hline AZ1 & $13.2 \pm 2.2$ & $14.0 \pm 0.0$ & $10.0 \pm 0.0$ & - & $9.2 \pm 6.0$ \\
\hline AZ2 & $18.0 \pm 0.8$ & $12 \pm 2.0$ & $10.0 \pm 0.0$ & - & $40 \pm 10$ \\
\hline AZ3 & $18.0 \pm 0.6$ & $10.6 \pm 4.0$ & $12.0 \pm 0.0$ & - & $17 \pm 6.8$ \\
\hline AZ4 & $8.0 \pm 3.4$ & - & $20.0 \pm 2.0$ & - & $19 \pm 7.0$ \\
\hline AZ5 & $6.6 \pm 2.9$ & $11 \pm 1.0$ & $19.0 \pm 3.0$ & - & $6.6 \pm 2.8$ \\
\hline AZ6 & $20.0 \pm 0$ & $9.2 \pm 4.0$ & $12.0 \pm 5.2$ & - & $20 \pm 8.4$ \\
\hline AZ7 & - & $13.2 \pm 5.6$ & $16.0 \pm 7.8$ & - & $15.0 \pm 5.0$ \\
\hline AZ8 & $15.0 \pm 3.0$ & $10.0 \pm 0.0$ & - & - & $26.0 \pm 6.0$ \\
\hline AZ9 & $15.0 \pm 5.0$ & $16.0 \pm 2.0$ & $19.0 \pm 5.0$ & - & $19.0 \pm 10$ \\
\hline Ciprofloxacin $(5 \mu \mathrm{g} / \mathrm{ml})$ & $28 \pm 8.2$ & $28 \pm 4$ & $28 \pm 4$ & $20 \pm 0$ & \\
\hline Fluconazole $(500 \mu \mathrm{g} / \mathrm{ml})$ & & & & & $54 \pm 18$ \\
\hline Negative control & - & - & - & - & - \\
\hline
\end{tabular}

Values are mean $\pm \mathrm{SD}(\mathrm{n}=3),(-)$ : no growth, AZ1: 2\{[4-(4-methylpiperazine-1-yl)but-2-yn-1-yl]sulfanyl\}-1,3-benzothiazole, AZ2: 2-\{[4-(2-methylpiperidine-1yl)but-2-yn-1-yl]sulfanyl $\}-1,3$-benzothiazole, AZ3: $2-\{[4-(2,6$-dimethylpiperidin-1-yl)but-2-yn-1-yl]sulfanyl $\}-1,3-b e n z o t h i a z o l e, \quad$ AZ4: $2-\{[4-(a z e p a n e-1-$ yl)but-2-yn-1-yl]sulfanyl\}-1,3-benzothiazole, AZ5: 2-\{[4-(pyrrolidin-1-yl)but-2-yn-1-yl]sulfanyl\}-1,3-benzothiazole, AZ6: 2-\{[4-(piperidin-1-yl)but-2-yn-1yl]sulfanyl\}-1,3-benzothiazole, AZ7: S-1,3-benzothiazol-yl-0-ethyl carbonothioate, AZ8: S-1,3-benzothizol-2-yl-0-methyl carbonothioate, AZ9: S-1,3benzothiazol-2-yl-0-(2-methyl propyl) carbonothioate. 
Table 2: Minimum inhibitory concentration (MIC) of compounds (AZ1-9) in $\mu \mathrm{g} / \mathrm{ml}$ against S. aureus, B. subtilis, E. coli, P. aeruginosa and C. albicans

\begin{tabular}{llllll}
\hline Compound & P. aeruginosa & E. coli & S. aureus & B. subtilis & C. albicans \\
\hline concentration & MIC/MBC $(\mu \mathrm{g} / \mathbf{m l})$ & MIC/MBC $(\mu \mathrm{g} / \mathbf{m l})$ & MIC/MBC $(\mu \mathrm{g} / \mathbf{m l})$ & MIC/MBC $(\mu \mathrm{g} / \mathbf{m l})$ & MIC/MBC $(\mu \mathrm{g} / \mathbf{m l})$ \\
\hline AZ1 & $62.5 / 250$ & $250 / 500$ & $125 / 500$ & $125 / 500$ & $125 / 500$ \\
AZ2 & $125 / 250$ & $250 / 500$ & $125 / 500$ & $62.5 / 125$ & $125 / 250$ \\
AZ3 & $125 / 250$ & $250 / 500$ & $125 / 500$ & $62.5 / 125$ & $125 / 250$ \\
AZ4 & $125 / 250$ & $125 / 250$ & $125 / 250$ & $62.5 / 125$ & $125 / 500$ \\
AZ5 & $125 / 250$ & $125 / 250$ & $125 / 250$ & $62.5 / 125$ & $62.5 / 250$ \\
AZ6 & $62.5 / 250$ & $250 / 500$ & $250 / 500$ & $125 / 250$ & $62.5 / 250$ \\
AZ7 & $62.5 / 250$ & $250 / 500$ & $250 / 500$ & $125 / 250$ & $62.5 / 125$ \\
AZ8 & $125 / 500$ & $250 / 500$ & $250 / 500$ & $125 / 250$ & $62.5 / 250$ \\
AZ9 & $62.5 / 250$ & 25 & $250 / 500$ & $125 / 250$ & $31.25 / 62.5$ \\
Ciprofloxacin & 50 & - & 50 & 25 & \\
Fluconazole & & - & - & - \\
Negative control & - & & & & \\
\hline
\end{tabular}

AZ1: $\quad 2\{[4-(4-m e t h y l p i p e r a z i n e-1-y l) b u t-2-y n-1-y l]$ sulfanyl $\}-1,3-b e n z o t h i a z o l e, \quad$ AZ2:2-\{[4-(2-methylpiperidine-1-yl)but-2-yn-1-yl]sulfanyl $\}-1,3-$ benzothiazole, AZ3:2-\{[4-(2,6-dimethylpiperidin-1-yl)but-2-yn-1-yl]sulfanyl\}-1,3-benzothiazole, AZ4: 2-\{[4-(azepane-1-yl)but-2-yn-1-yl]sulfanyl\}1,3-benzothiazole, AZ5: 2-\{[4-(pyrrolidin-1-yl)but-2-yn-1-yl]sulfanyl\}-1,3-benzothiazole, AZ6: 2-\{[4-(piperidin-1-yl)but-2-yn-1-yl]sulfanyl\}-1,3benzothiazole, AZ7: S-1,3-benzothiazol-yl-0-ethyl carbonothioate, AZ8: S-1,3-benzothizol-2-yl-0-methyl carbonothioate, AZ9: S-1,3-benzothiazol-2yl-0-(2-methyl propyl) carbonothioate, MIC: Minimum Inhibitory Concentration,MBC: Minimum Bactericidal Concentration, MFC: Minimum Fungicidal Concentration.

\section{DISCUSSION}

\section{Chemistry}

The designed compounds were prepared as shown in schemes $(1,2$ and 3). (Scheme 1) involved the alkylation of 2-mercaptobenzothiazole with 3-bromo prop-1-yne (propargyl bromide) in the presence of acetonitrile as a solvent, under basic conditions. The reaction involves direct displacement of the anionic sulfur (thiolate anion) in the thiazole ring on the propargyl bromide, to generate 2(prop-2-yn-1-ylsulfunyl)-1, 3-benzothiazole AZ0. The Mannich reaction of 2-(prop-2-yn-1-ylsulfanyl)-1,3-benzothiazole AZ0, Paraformaldehyde, appropriate cyclic amine, and a catalytic amount of cuprous chloride in peroxide-free 1,4-dioxane was heated to 70 $75^{\circ} \mathrm{C}$ to yield the designed compounds (AZ1-6).

The yield obtained ranged from 42.47 to $100 \%$. The proposed mechanism for Mannich reaction is shown in (Scheme 2). In order for Mannich reaction to proceed, a reactive immonium cations intermediates should be formed, from the condensation of the formaldehyde and the appropriate amines (Schiff base formation). The attack of the carbanion in 2-(prop-2-yn-1-ylsulfanyl)-1,3benzothiazole cuprous salt on the Schiff base generates the desired Mannich products (AZ1-6). The UV-HPLC, FT-IR, ${ }^{1} \mathrm{H}-\mathrm{NMR}$, ${ }^{13} \mathrm{C}$-NMR, DSC and elemental analysis were consistent with the assigned structures. (Scheme 3) involved the addition of chloroformate to the 2-MBT, in the presence of absolute ethanol as a solvent under basic conditions. The reaction involves direct displacement of the anion sulfur in the thiazole ring on the ethyl, methyl or isobutyl chloroformate to generate S-1, 3-benzothiazole2-yl-0-t carbonothioate (AZ7-9). The yield obtained ranged from 51 to $83 \%$
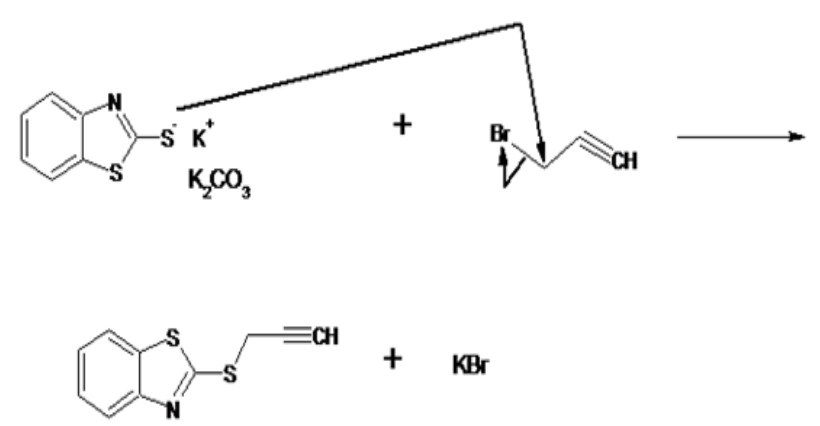

2-(Prop-2-yn-1-ylsulfanyl)-1,3-benzothiazole

Scheme 1: Alkylation Reaction of 2-MBT
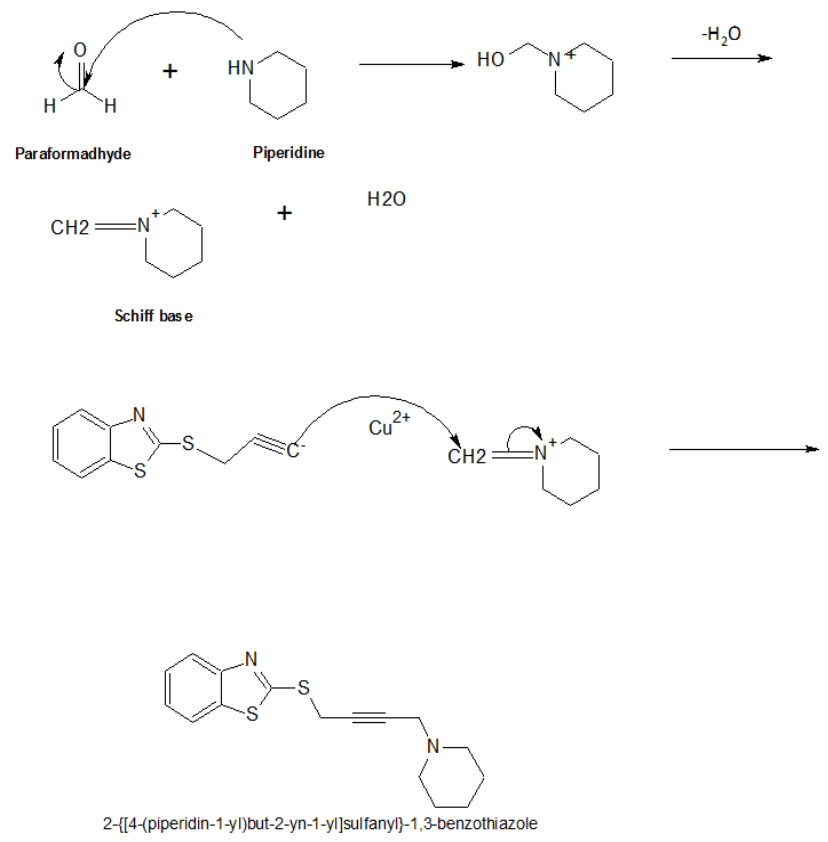

Scheme 2: Mannich reaction proposed

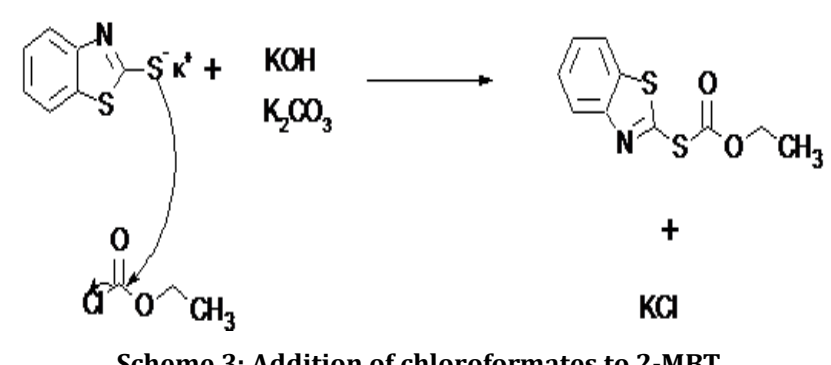

Scheme 3: Addition of chloroformates to 2-MBT

In order to determine the antimicrobial activity of the newly synthesized compounds, agar diffusion method, and broth dilution method were the most common methods used in evaluating the antimicrobial activity $[12,13]$. The variability of the results using the agar diffusion method, and broth dilution method may be due to the high molecular weight of the of $2-\{[4-(\mathrm{t}$-amino-1-yl)but-2-yn-1-yl] 
sulfanyl\}-1,3-benzothiazole derivatives (AZ1-6), and S-1,3benzothiazol-2-yl-0-t-carbonate derivatives (AZ7-9), and their low solubility in water made their diffusion in agar medium is slow, giving zone of inhibition which reported as low antimicrobial activity. The good activity of (AZ1, AZ6, AZ7 and AZ9) against P. aeruginosa may be attributed to the diffusion of the compound, using both lipid-mediated and a porins-mediated pathway for entry into the bacterial cells. The reasons for the entry of these compounds to P. aeruginosa by dual pathway may due to the drug flux and the susceptibility, both are sensitive to the presence of porins (in particular OmpF), and to the manipulations that disrupt the outer membrane LPS barrier, through electronegativity overlap. In addition, the relative contribution of the two pathways may correlate with the hydrophobicity of these compounds, due to the presence of aryl moiety and the protonation state of these compounds, due to the presence of cyclic amine moiety or COOR moiety [14]. AZ4 is a more lipophilic compound than other compounds that affect $\mathrm{P}$. aeruginosa (like AZ1, AZ6, AZ7 and AZ9), due to azepane cyclic amine has 6 carbons in its structure. E. coli strains have a lacking in pore-forming proteins especially $\mathrm{OmpF}$ [15], so the only way to enter bacteria cell is the permeation lipid bilayer by high lipophilic compounds like AZ4. From the previous results, the newly synthesised compound showed higher antibacterial activity against P. aeruginosa than against E. coli. The newly synthesized compounds are active against Gram-positive bacteria, that is attributed to the peptidoglycan of Gram-positive cells is more receptive to antimicrobials, due to the absence of the outer membrane. The antibacterial activity of AZ2 (2-methyl piperidine), AZ3 (2,6-dimethyl piperidine), AZ4 (azepane) and AZ5 (pyrolidine) against Gram-positive bacteria is attributed to the lipophilicity and steric effect, according to the large size of 2-methylpiperidine, 2,6dimethylpiperidine, azepane and pyrolidine respectively. Lipophilicity may facilitate the diffusion of compounds through the cell wall, and the steric effect may exert physical pressure on the cell wall of Grampositive bacteria. The newly synthesised compounds have antimicrobial activity against B. subtilis more than antimicrobial activity against $S$. aureus.

\section{CONCLUSION}

In conclusion, we have reported the synthesis of novel series of aminoacetylenic, and carbonothioate 2-mercaptobenzothiazole derivatives. A unique and new amino acetylenic side chain that provides additional forces of interaction with the microorganism. Data on antibacterial and antifungal activity generated from this investigation, merit the generation of new amino acetylenic and carbonothioate 2mercaptobenzothiazole derivatives, with more potent antimicrobial activity through changes in lipophilicity and electronegativity.

\section{ACKNOWLEDGMENT}

The authors would like to thank the University of Petra/Faculty of Pharmacy for providing the necessary facilities to carry out this work.

\section{CONFLICT OF INTERESTS}

The authors have declared no conflict of interest.

\section{REFERENCES}

1. Kumar K, Awasthi D, Lee SY, Zanardi I, Ruzsicska B, Knudson S, et al. Novel trisubstituted benzimidazoles, targeting Mtb FtsZ, as a new class of antitubercular agents. J Med Chem 2011;54:374-81.

2. Keri R, Patil MR, Patil SA, Budagumpi S. A comprehensive review in current developments of benzothiazole based molecules in medicinal chemistry. Eur J Med Chem 2015;89:207-51.

3. Dancer SJ. Proceeding of the lancet conference on healthcareassociated infections London UK. The role of environmental cleaning in the control of hospital-acquired infection. JHI 2009;73:378-85.

4. Sasikumari S, Haripriya M, Anjali T. Synthesis and evaluation of anti-inflammatory and antibacterial activities of some 1, 2benzisoxazole derivatives. Int J Chem Pharm Res 2016;8:64-7.

5. Azam MA, Suresh B. Biological activities of 2mercaptobenzothiazole derivatives: a review. Sci Pharm 2012;80:789-823.

6. Khokra SL, Arora K, Mehta H, Aggarwal A, Yadav M. ChemInform abstract common methods to synthesise benzothiazole derivatives and their medicinal significance. Chem Inform 2011;42. Doi:10.1002/chin.201142237

7. Ali, Siddiqui N, Khan SA, Haque SE, Bhat MA. $N$-[(6-Substituted1,3-benzothiazole-2-yl)amino]carbonothioyl-2/4-substituted benzamides: synthesis and pharmacological evaluation. Eur J Med Chem 2008;43:1114-22.

8. Ali S. Synthesis and preliminary antibacterial study of new 2mercapto-1,3-benzothiazole derivatives with expected biological activity. Afr J Plant Sci 2013;13:119-24.

9. El-Shaaer FP, Lacova M, Chovancova J, Stankovicova H. Synthesis, antimicrobial activity and bleaching effect of some reaction products of 4-oxa-4H-benzopyran-3-carboxaldehydes with amino benzothiazoles and hydrazides. Farmaco 199;53:224-32.

10. Desai KG. Rapid and efficient synthesis of some active biological 2-azetidinones under microwave irradiation. Indian J Chem 2005;44B:2093-6.

11. Yilmaz S. Synthesis and in vitro antimicrobial activity of novel 2-(4-substituted-carboxamido) benzyl/phenyl) benzothiazoles. Croat Chem Acta 2013;86:223-31.

12. Tortora GJ, Funke BR, Case CL. Microbiology: an introduction. $11^{\text {th }}$ ed. San Francisco (CA) USA, Pearson Benjamin Cummings publishers; 2013.

13. Patil NR, Gadagil SA. Performance of CHROM agar medium and conventional methods for detection of methicillin-resistant Staphylococcus aureus. Asian J Pharm Clin Res 2016;9:136-9.

14. Jayaprakash R, Saroj Kumar SHA, Hemalatha S, Easwaramoorthy D. Synthesis, characterization, quantitative structure-activity relationship, docking, antibacterial activity, and brine shrimp lethal, studies on L-phenylalanine Schiff bases. Asian J Pharm Clin Res 2016;9:1-6.

15. Cohen SP, Hooper DC, Wolfson JS, Souza KS, McMurry LM, Levy SB. Endogenous active efflux of norfloxacin in susceptible Escherichia coli. Antimicrob Agents Chemother 1988;32:1187-91.

\section{How to cite this article}

- Aseel Alsarahni, Zuhair Muhi-Eldeen, Elham Al-Kaissi, Ibrahim Al-Adham, Najah Al-Muhtaseb. Synthesis and structural elucidation of amino acetylenic and thiocarbamates derivatives for 2-mercaptobenzothiazole as antimicrobial agents. Int J Pharm Pharm Sci 2017;9(2):192-197. 